\title{
Geschichte der Medizin.
}

\section{Alexander v. Humboldt und Robert Remak. Von G. Mamlock.}

Am 10. November des Jahres 1856 teilte Alexander v. Hu mboldt dem Generaldirektor der Königlichen Museen in Berlin Ig naz v. Olfers mit, daß ihm bei der Besichtigung einer Bildersammlung ein Bild auf den Kopf gefallen wäre, wobei gleichzeitig der neben ihm stehende „elektromagnetische Dr. Remak“ unbedeutend verletzt wurdel).

Der hier Genannte war der 1815 geborene Dr. Robert Remak, Vater des jüngst verstorbenen Neurologen.

Seit 1838, wo Remak seine Dissertation') an Alexander v. Hu m. boldt gesandt hatte, interessierte sich dieser für ihn und suchte ihm bei einer Universitätsstellung behilflich zu sein. Die hierüber geführte Korrespondenz hat soeben L. Geiger mitgeteilt ${ }^{3}$ ). Die Briefe $\mathrm{Hu}$ mboldts an Remak gewähren einen lehrreichen Blick in die Anfänge der Berliner Medizinischen Fakultät.

Diese befand sich damals in einem Erneuerungsstadium, und zwar wirkten in den theoretischen Fächern Johannes Müller, in den klinischen Schönlein als Fermente.

Dieser hatte durch Neuordnung des Unterrichts und Einführung der deutschen Sprache beim. Vortrag die Ausbildungsmöglichkeiten bedeutend gefördert. Bereits bei seiner Berufung aus Zürich hatte er nach dieser Richtung bestimmte Zusicherungen von der preußischen Regierung verlangt. Er hatte dem preußischen Unterrichtsminister am 4. Mai 1839 Folgendes geschrieben ${ }^{4}$ ):

$\mathrm{Z}$ ü ri ch, 4 V: 1839

Je ehrenvoller der Ruf ist, den Ihre Exz. durch Zuschrift vom 16. vor. $M$ an mich gelangen ließen. je glănzender der Wirkungskreis erscheint, der mir dadurch eröffnet wird, Je gröBer ich das Glick erachte, dadurch in die Dienste durch lebhafter fähle ich aber auch die Verpflichtung, vor Abgabe meiner gehorsamsten
Erklärung streng und gewissenhaft meine Befähiguna. aufmerksam und genau alle Umstände und Verhältnisse zu proffen. Für die Stellung, die Besoldung und die sonstigen Emolumente, die $\mathrm{S}$. Majestät mir gnädigst anzubieten geruhten, kann ich nur den innigsten Dank aussprechen. Dagegen wage ich gehorsamst einige Bitten anzubringen und diese der huldvollen Berücksichtigung Ihre Exz. um so dringender $z u$ empfehlen, als ich die Ueberzeugung hege, dąß von derer gnädigen Gewährung zunächst der Erfolg meiner Bemühung als klinischer Lehrer bedingt werde. Die eine dieser Bitten bezieht sich auf die genaue Regulierung bedingt werde. Die eine dieser Bitten bezeht den Aerzten des Charité-K $K_{\text {ankenhauses }}$ in der Art, daB mir dadurch die 'Möglichkeit geboten werde, die für den klinischen Unterricht bestimmten Betten stets mit instruktiven Krankheitsfällen, teils durch freie Auswahl aus den in den verschiedenen Abteilungen des genannten Kranken-

1) Briefe Alexander v. Humboldts an Ignaz v. Olfers. Heraus. cenon Gon Gan-Rat Dr. E. W. M. V. O Ifers (Königsberg). Nürnberg-Leipzig bei W. E. Sebald. S. 211 Nr. 298. - 2) Observationes anatomicae et microscopicae de systematis nervosi structura. $-{ }^{3}$ ) Jahrb. f. jüdische Geschichte u. Literatur. 1916. - ${ }^{4}$ ) Original, wie auch des folgenden Briefes in der Kgl. Bibliothek in Berlin. 
hauses befindllchen Patienten, teils durch die ungehinderte Zurückgabe der für den Unterricht fernerhin untaugllchen Kranken, zu belegen.

Meine andere gehorsamste Bitte geht dahin, mir gnädigst gestatten zu wollen, den klinischen Unterricht in deutscher Sprache zu erteilen. Ich werde mlch nicht unterfangen, beurteilen $z u$ wollen, in wiefern die Vorschrift, daß der klinische Unterricht an den Universitäten in lateinischer Sprache gegeben werdèn solle, mit dem System des Unterrichts zusammenhängend und von höheren Răckslchten geboten, die erbetene Abänderung zulasse oder nicht; ich erlaube mir nur, meine Bitte durch die Ueberzeugung zu motivieren, daß die Aufgabe, die Lehren der Arzneikunde In lateinischer Sprache vorzutragen, bei dem gegenwärtigen Stande dieser Wissenschaft und bei ihrer unter dem'Einflusse der übrigen Naturwissenschaften rasch fortschreitenden Entwicklung nicht nur eine sehr schwierige, sondern nahezu unlösbare sei.

Ich wünsche nichts sehnlicher, als daß es Ihrer Exz. gefallen möge, durch die gnädige Berücksichtıgung meiner gehorsamsten Bitten die Hindernisse zu entfernen, dle mir über die Möglichkeit eines crfolgreichen Wirkens als klinischer Lehrer zu Berlin große Zweifel erregen. Sobald Ihrer Exz. desfallige Entschließung an mich gelangen wird, werde ich sogleich alle Anstalten treffen, um mit künftigem Semester die mir gnädigst übertragene Stelle antreten zu können; da ich aus dem diesseitigen Staatsdienst ohnehin erst mit Ende des Sommersemesters entlassen werden kann, indem ein Gesetz den Austritt aus dem Universitätsverbande während der Dauer des Semestralkurses untersagt.

Noch ein zweites - meines Wissens bisher unbekanntes Schreiben Schönleins ist bezeichnend für seinen Reformeifer: suchte er doch der Pathologischen Anatomie den ihr gebührenden Rang im Universitätsunterricht zu verschaffen. Er gab am 18. August 1855 das folgende Gutachten ab:

Da der Hauptgrund, welcher bisher der Gewährung der Bitte des H. D. Dubois ${ }^{1}$ ) um eine außerordentliche Professur an der Friedrich-Wilhelms-Universităt zu Berlin entgegenstand - nämlich der, daß er bis jetzt seine Lehrbefähigung noch nicht durch Vorlesungen an der Universität nachgewiesen habe - beseitigt ist und auch der Ordinarius der Physiologie Herr Dr. Müller die Ernennung eines Extraordinarius für das gleiche Fach befüwortet hat; so möchte kaum mehr ein realer oder persönlicher Grund geltend gemacht werden können, weshalb Herr Dubois abschlägig zu bescheiden.

Ich kann diese Gelegenheit aber nicht unbenutzt vorübergehen lassen, ohne das hohe Ministerium auf einen Mann aufmerksam zu machen, der diese Auszeichnung - die Ernennung zum außerordentlichen Professor in der medizinischen Fakultăt - mir noch in weit höherem Maße zu verdienen scheint als Herr Dubois; denn die Physiologie ist durch $\mathrm{J}$. Mäller so glänzend und erfolgreich vertreten, daß es wahrlich elnes Suppleanten nicht bedarf, während dagegen die pathologische Anatomie dieser so wichtige Zweig in der Entwicklung der neuen Medizin, nur durch $M e c k l^{2}$ ), und zwar durch Lehre und Schrift in gleicher Auszeichnung kultiviert wird. S chönle in.

In die Zeit zwischen diese beiden Schreiben fallen die von Geiger veröffentlichten. Sie sind ein neuer Beweis dafür, daß Hu mbold̀t bei jeder Gelegenheit bestrebt war, die ,verwahrloste, durch den Uebermut der Spekulation fast erdrückte Naturforschung wieder zu Ehren zu bringen ${ }^{63}$ ). Von diesem Gesichtspunkte aus mußte ein Forscher wie Remak besondere Aufmerksamkeit erregen.

Für den Mediziner ist Geigers Veröffentlichung - in der allerdings die ,gelehrten Exkurse den Text überwuchern") “ - in erster Reihe in teressant.

Bei der Aussichtslosigkeit für einen Juden, damals in Preußen eine Dozentur zu erreichen, wandte sich $\mathrm{Hu}$ mboldt zunächst an den ihm befreundeten Präsidenten der Petersburger Akademie, Uwar of $f^{5}$ ). In einem aus Sans-Souci, 8. September 1839 datierten Schreiben empfiehlt er Remak angelegentlichst wegen seiner Arbeiten über die feinere Anatomie und Physiologie des Zentralnervensystems. Hu mboldt schreibt weiter, er habe mit Ehrenberg) die Remakschen Befunde mikroskopisch nachgeprüft und könne sie nur durchaus anierkennen. Auch sei Johannes Müller bereit, die Empfehlungen aufs wärmste zu unterstützen. Unter Hinweis auf Re maks zahlreiche in deutscher, Iateinischer und polnischer Sprache veröffentlichten Arbeiten empfiehlt $\mathrm{Hu}$ mboldt seinen Schützling für eine freigewordene Professur für Physiologie in Wilna. Natürlich vergeblich.

Von einer seitens Remaks beabsichtigten Bewerbung in Paris riet Humboldt gänzlich ab, dagegen verwandte er sich um so energischer bei Friedrich Wilhelm IV. Die Schwierigkeiten, die ihm auf dem bekannten ,Instanzenwege" entgegentraten, waren nicht gering. $\mathrm{Hu} \mathrm{m}$ boldt korrespondierte abwechseInd mit den behördlichen Organen und

1) Fmil du Bois Reymond - $\left.{ }^{2}\right)$ H Meckel v. Hemsbach, 1821-56, Virchows Vorgänger in der Charité-Prosektur von 1852-56. a) Treitschke. Deutsche Geschichte im Neunzehnten Jahrbundert. III. Teil. 6. Aufl. Leipzig 1908 S. 432 - 4) Geigers Worte über Steinschneiders Publikationen. - Die Ueberladung mit teilweise nicht hingehörigen gelehrten Ausführungen erschwert die Lektüre der Publikation Geigers außerordentlich und läßt stellenweise die Briefe in den Hintergrund treten. - 5) Dieser ,, Minister für Volksaufklärung، hat die drei Schlagworte: „Orthodoxie, Selbstherrschaft und Nationalismus"' geprägt, die als Richtschnur für das geistige und politische Leben Rů lands maßgebend wurden. (Vgl. Th. Schiemann, Russische Köpfe Berlin 1916. S. 214.) $-{ }^{6}$ ) Mediziner und Naturforscher, begleitete $\mathrm{H} \mathrm{u} \mathrm{m}$. bold t 182? nach Asien. Seit 1839 bis 1876 Professor für Medizin in Berlin. gab Remak Bescheid, wenn or an irgendeiner Stelle auf ,intolerante Gründe" stieß. Unter diesen Umständen versuchte $\mathrm{Hu} \mathrm{m}$ bold $\mathrm{t}$ durch Vorstellung bei Friedrich Wilhelm IV. etwas zu erreichen, und er veranlaßte Remak, der seit 1843 Schönleins Assistent war, zu einer Eingabe unmittelbar an den König; ihr sollte er seine beiden neuesten und bedeutendsten Werke beilegen.

Das eine war: „Diagnostische und pathogenetische Untersuchungen in der Klinik des Geh.-Rats Dr. Schönlein, auf dessen Veranlassung angestellt und mit Benutzung anderweiter Beobáchtungen veröffentlicht" (mit 1 Kupferstich, Berlin 1845, Hirschwald). Das andere war die H u mbold t gewidmete Schrift „Ueber ein selbständiges Darmnervensystem" (Berlin 1847).

Hu mbold ts Bitte, das Werk gleichzeitig Schönlein und J. Müller zu widmen, erfüllte Remak nur bezüglich des letzteren. Ein Grund dafür ist nicht ersichtlich. Vielleicht gibt eine Bemerkung $\mathbf{R u d o l f}$ Virchows in einem Brief an seinen Schwiegervater, 15. Februar 1856, aus Würzburg den Aufschlu $B^{1}$ ). Er erwähnt darin seine mögliche Berufung nach Berlin und scheint an eme ernsthafte Konkurrenz , von seiten Remaks gedacht zu haben. Denn er beschwichtigt sich gewissermaßen selbst mit den Worten: „Schönlein wird nicht zu eifrig für Remak sein; ich kenne das von Alters her: Er hat schon früher Remak gegen mich postiert $t^{2}$ ) und ihn endlich doch fallen lassen \%

Möglicherweise steht damit in Zusammenhang die in Hu mboldts Brief vom 21. Juni 1847 angedeutete Trennung Rema ks von Schönlein.

Humboldt hielt aber trotz aller seiner Bemühungen es für angebracht, eine „Rückversicherung" einzugehen, und so wandte er sich am 27. April 1846 mit einem sehr warmen Empfehlungsschreiben an J. v. Liebig ${ }^{3}$, der damals in Gießen allmächtig war.

„Dle Professur in der medizinischen Klinik ist bel Ihnen noch immer unbesetzt. Ein laberaus tätiger, talentvoller, mit mir befreundeter Junger Mann, Dr. Remak, vereinigt in merkwürdiger Weise eine ausgezeichnete klinische Praxis (und eine Praxis unter den Augen unseres Schönlein 1) mit einer glücklichen Vorliebe für die feinste mikroskopische Zergliederung belebtér Organismen. Johannes $M$ ü 11 er und $S c h b n l e l n$ werden über thn ebenso günstig berichten, als lch. Bei den vlelen herrlichen physiologischen Aussichten. dle Sie, lieber Freund, eröffnen, müßte die Nähe des Dr. Remak Ihnen ja willkommen sein, und die noch immer bestehende Intoleranz unserer Gesetzgebung wird, wenn Sie dem Manne Ihren Schutz verleihen, fa wohl meinem Wunsch nicht entgegenstehen."

Inzwischen hatten $\mathrm{Humboldts}$ Bemühungen beim König Erfolg gehabt, und im Jahre 1847 konnte sich Remak in Berlin habilitieren. H u mbold t hat während der ganzen Jahre Re mak nicht nur mit seiner überlegenen Diplomatie beratend zur Seite gestanden, sondern auch seine Arbeiten kritisch begutachtet. Die bis zum Jahre 1857 reichenden Briefe bekunden $\mathrm{Hu}$ mbold ts uneingeschränkte Anerkennung für Remaks anatomische und physiologische Untersuchungen, und es ist noch heute an den Urteilen Hu mboldts der frische Eindruck zu erkennen, den Remaks originelle Forschungen auf die vormärzliche Gelehrtenwelt machten.

Auch Remak dürte das Wort des Ovid auf sich beziehen: „Principibus placuisse viris non ultima laus est." 